\title{
Chitosan Nanocomposites with Graphene-Based Filler
}

\author{
Thuany Garcia Maraschin ${ }^{a}$, Roberto da Silva Correa ${ }^{b}$, Luiz Frederico Rodrigues, , Naira Maria Balzarettid ${ }^{d}$,

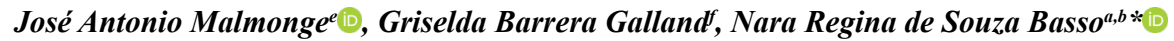 \\ aPontifícia Universidade Católica do Rio Grande do Sul - PUCRS, Escola Politécnica, Av. Ipiranga, 6681, \\ 90619-900, Porto Alegre, RS, Brasil. \\ ${ }^{b}$ Pontifícia Universidade Católica do Rio Grande do Sul-PUCRS, Escola de Ciências, Av. Ipiranga, 6681, \\ 90619-900, Porto Alegre, RS, Brasil. \\ 'Instituto do Petróleo e dos Recursos Naturais - IPR, Av. Ipiranga, 6681 Prédio 96J, 90619-900, \\ Porto Alegre, RS, Brasil. \\ ${ }^{d}$ Universidade Federal do Rio Grande do Sul - UFRGS, Instituto de Física, Av. Bento Gonçalves, 9500, \\ 91570-970, Porto Alegre, RS, Brasil. \\ eUniversidade Estadual Paulista, UNESP, Faculdade de Engenharia, Avenida Brasil, 56, 15385-000, \\ Ilha Solteira, SP, Brasil.

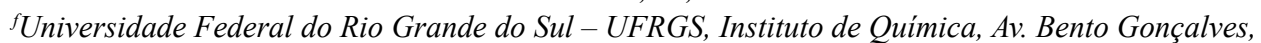 \\ 9500, 91570-970, Porto Alegre, RS, Brasil.
}

Received: January 7, 2019; Revised: November 1, 2019; Accepted: December 19, 2019

This study evaluates the properties of chitosan (CS) membranes modified with different percentages $(0.5 \%, 3 \%$, and $5 \% \mathrm{w} / \mathrm{w})$ of a graphene-based material. Graphene oxide $(\mathrm{GO})$ and reduced graphene oxide (RGO) were obtained by the chemical exfoliation of graphite and thermal reduction. Then, they were characterized by electrical conductivity measurements, FESEM, XRD, AFM, and Raman spectroscopy. The composites' morphology was evaluated by FESEM. The degree of swelling over a $48 \mathrm{~h}$ period and mass loss behavior in phosphate-buffered saline solution for up to 70 days were also studied. The hydrophilicity of the CS and CS/graphene nanocomposites was examined by water contact angle. The graphene materials showed small stacks (6-8 sheets) with low defect density and nanoscale thickness $(1.3-5.9 \mathrm{~nm})$. The dispersion of the graphene material in the CS matrix significantly decreased the degree of swelling $(460 \%)$ but did not modify the hydrolytic degradation process and the hydrophilicity of membranes.

Keywords: Graphene oxide; reduced graphene oxide, chitosan; biodegradable polymer.

\section{Introduction}

Graphene consists of a single layer of graphite with a hexagonal carbon aromatic structure' ${ }^{1}$. Owing to its unique conjugated structure, large surface area, and relatively low cost, graphene has found novel in vitro and in vivo applications in the field of pharmacology ${ }^{2}$, It also holds great promise for applications in fields like gene and drug delivery, tissue engineering, and cancer therapy ${ }^{3}$.

Flake-type graphene can be prepared by a top-down method from graphite because graphite consists of a stack of flat graphene layers and is readily available and cheap. Graphene can be peeled mechanically from graphite; however, this method is not suitable for large-scale graphene production owing to its low productivity. Pristine graphene has low dispersibility in water and in polymer matrices. However, its functionalized form with epoxy, hydroxyl, and carboxyl functional groups on its surface shows improved processability and dispersibility. The polar interaction between functional graphene and organic polymers results in better filler dispersion ${ }^{4-7}$.
Therefore, structures derived from graphene, such as graphene oxide (GO) and reduced graphene oxide (RGO), have attracted increased interest. The chemical method is popularly used for synthesizing graphene from graphite by producing a colloidal suspension ${ }^{8}$. Although increasing the number of oxygen-containing groups improves the aqueous dispersibility, it also increases defects in the structure, making it electrically insulating. To restore the conductivity, the functional groups in GO must be partially removed by thermic reduction. This method is economical and eco-friendly because it does not use any solvent ${ }^{7,9}$, and as a result, reduced graphene oxide with a few layers can be obtained.

Graphene-like materials such as GO and its reduced form, RGO, are promising for the development of antibacterial surfaces owing to their biocidal ${ }^{10}$, antirust ${ }^{11}$ and antibacterial ${ }^{12}$ activities. It was used as a support to disperse and stabilize various nanomaterials such as metals, metal oxides, and polymers with high antibacterial efficiency. GO and RGO can be well dispersed to produce thin sheets that wrap bacteria easily and then inactivate the bacteria through the membrane stress induced by the sharp edges and oxidative stress induced by the basal planes ${ }^{12}$. 
The linkage of graphene with polysaccharides (such as starch, cellulose, and chitosan) results in nanocomposites with improved mechanical properties. Mixed matrix membranes hybridized by incorporating nanoparticles have attracted considerable attention owing to the intriguing potential of developing novel membranes with multiple functionalities ${ }^{13}$. The use of these biomaterials has lower environmental impact compared to the use of nanocomposite matrices from oil and can promote lowcost biodegradable materials, being a source of important materials for polymeric membranes and enabling the reuse of a large volume of residues ${ }^{14-17}$.

Chitosan (CS) is a biopolymer that is produced by chitin deacetylation. Flexible CS membranes can be used as artificial skin and are being tested for treating burnt skin; they are proving to be efficient compared to gauze for such treatment. CS membranes contain proteins that promote healing; further, the CS structure assists in cell growth and tissue organization $^{18,19}$. Despite the biocompatibility of the pristine CS matrix, CS may show reduced performance in extended use owing to its swelling and degradation characteristics ${ }^{20}$. To avoid this issue, various methods such as blending and crosslinking have been tested. In addition, the development of membranes containing nanofillers has been investigated to improve the physical and chemical properties ${ }^{20,21}$.

The functional groups of GO and RGO enable reactions with amine or hydroxyl groups to form amide or ester linkages. Therefore, $\mathrm{CS}$ can be grafted by amide bonds between the reactive groups of CS and the carboxyl groups present in GO and RGO. In addition, functional groups attached to graphene surfaces can improve interfacial interactions with the polymeric matrix ${ }^{22,23}$.

Hegab et $\mathrm{al}^{10}$ demonstrated that the antibacterial and antifouling properties of CS membranes can be enhanced by binding graphene-based materials to the membrane surface. The antibacterial efficiency of these polymeric graphene membranes ranged from $64 \%$ to $99 \%$. Others studies demonstrated the use of CS membranes with graphene for applications such as water purification, tissue engineering, and food packaging ${ }^{6,24}$.

Despite the biocompatibility of the CS matrix and its potential for tissue regeneration, the mechanical properties and biological response of these matrices are considered inadequate for bioengineering. The difficulties faced include degradation control and the swelling of pure CS matrices ${ }^{20}$. In this study, a $\mathrm{CS}$ matrix containing different percentages of GO and RGO was examined using field-emission scanning electron microscopy (FESEM), X-ray diffraction (XRD), atomic force microscopy (AFM), Raman spectroscopy, and electrical conductivity measurements. The hydrolytic degradation, degree of swelling in graphene/CS membranes and the hydrophilicity of membranes was also evaluated.

\section{Experimental Section}

\subsection{Materials and preparation}

All the chemicals were used as received without any further purification: Acetic Acid (99\%, NEON), Hydrochloric Acid (37\%, MERCK), Nitric Acid (65\%, Química Moderna), Sulfuric Acid (97\%, Química Moderna), Potassium chlorate (PA, VETEC), Phosphate buffered saline (Aldrich, 10\% Conc). The raw Grafine 99200 (FINE) was provided by Nacional de Grafite.

\section{Graphene oxide and reduced graphene oxide preparation}

The graphene oxide (GO) was synthesized from FINE graphite powder using the modified Staudenmaier

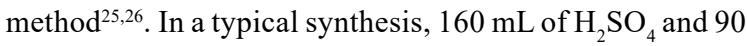
$\mathrm{mL}$ of $\mathrm{HNO}_{3}$ were stirred in a round-bottomed flask placed in an ice bath for $1 \mathrm{~h}$. After this time, $10 \mathrm{~g}$ of graphite FINE was added and stirred for $20 \mathrm{~min}$. Subsequently, $110 \mathrm{~g}$ of $\mathrm{KClO}_{3}$ was added slowly over 4 hours, with the temperature being controlled to prevent a rise above $10^{\circ} \mathrm{C}$. The reaction proceeded for 24 hours. On completion of the reaction, aqueous $\mathrm{HCl}$ solution $(10 \% \mathrm{v} / \mathrm{v})$ was added to the suspension in order to remove sulfate ions. Afterwards, washing with deionized water and centrifuging (5000 $\mathrm{rpm}$ ) were carried out several times in order to reach $\mathrm{pH}$ 3. The graphite oxide suspension was sonicated for 4 hours and the resulting suspension of graphene oxide (GO) was placed in a dialysis membrane in order to reach $\mathrm{pH} 5$, and then the resulting brown suspension was dried in an oven at $150{ }^{\circ} \mathrm{C}$. The $\mathrm{GO}$ resulting powder was heated $1000{ }^{\circ} \mathrm{C}$ for $30 \mathrm{~s}$ in an oven, using a quartz ampoule generating the reduced graphene oxide $(\mathrm{RGO})$.

\section{Preparation of chitosan membranes}

Chitosan powder was obtained from Sigma-Aldrich (degree of deacetylation: $85 \%$, Mw $500.000 \mathrm{~g} / \mathrm{mol}$ ). Chitosan membranes were prepared by solvent casting according to following procedure: $0.4 \mathrm{~g}$ of chitosan (CS) was suspended in $20 \mathrm{~mL}$ of acetic acid solution $(1 \% \mathrm{v} / \mathrm{v})$ and stirring for 5 minutes at room temperature, thereafter the suspension was subjected to sonication probe (Ultronique QR500, $40 \mathrm{kHz}$ ) for 9 minutes until the mixture became thick viscous liquid. Finally, the suspension was cast onto polystyrene dish (9 $\mathrm{cm}$ diameter) and allowed to dry conventional oven at $50{ }^{\circ} \mathrm{C}$ for 12 hours.

$\mathrm{CS}$ is only soluble in an aqueous medium in the presence of a small amount of acid such as $\mathrm{AcOH}$. For biomedical applications, the membrane surface must be neutralized ${ }^{27}$. For this purpose, the membranes were washed with $1 \%$ sodium hydroxide solution (w/v) and dried for $24 \mathrm{~h}$ at $50^{\circ} \mathrm{C}$. 


\section{Production of nanocomposites membranes}

The GO and RGO powder were dispersed into $20 \mathrm{~mL} 1 \%$ $(\mathrm{v} / \mathrm{v})$ acetic acid solution by sonication probe for 9 minutes at room temperature. Subsequently, $0.4 \mathrm{~g}$ chitosan powder was added into the suspension under stirring for 5 minutes, thereafter the suspension was subjected to sonication probe for another 9 minutes until the mixture became thick viscous liquid. The same procedure described in the previous item was used to remove the solvent and to wash the nanocomposites membranes surfaces. It must also be taken in consideration in this work that besides the presence of oxygenated anchoring sites in both materials based on carbon, the charge dispersion was facilitated by sonication probe. Both procedures guaranteed adequate charge dispersion in the polymeric matrix.

Nanocomposites membranes with $0.5 \mathrm{wt} \%, 3.0 \mathrm{wt} \%$ and $5.0 \mathrm{wt} \%$ GO loadings were coded as $\mathrm{CS} / \mathrm{GO} 0.5, \mathrm{CS} /$ $\mathrm{GO} 3$ and $\mathrm{CS} / \mathrm{GO} 5$ respectively. Likewise, membranes with RGO were coded as CS/RGO0.5, CS/RGO3 and CS/RGO5.

\subsection{Characterization of $G O$ and $R G O$ sheets and $C S /$ Graphene membranes}

\subsubsection{Morphology and structure}

The morphology of GO and RGO sheets, CS membrane, and $\mathrm{CS} /$ Graphene nanocomposites membranes were assessed using Scanning Electron Microscopy (FESEM) with Inspect F50 microscope. FESEM was also used to measure the thickness of all membranes by xTm, FEI software. The surface morphology and roughness GO and RGO were also evaluated by atomic force microscope (AFM), with Dimension Icon PT microscope, Bruker. The samples were deposited on Si substrate. The height (in $\mathrm{nm}$ ) of the estimated stacks of graphene sheets was obtained by the NanoScope program. Transmission electron microscopy (TEM) analyzes were obtained by Microscope EOL, 2010 operating at 200 $\mathrm{kV}$, samples were prepared in an appropriate solvent. The sample with a concentration of approximately $0.1 \mathrm{mg} / \mathrm{mL}$ was deposited on a 300 mesh copper grid.

The structures of the GO and RGO were analyzed by X-ray diffraction (XRD) performed on a diffractometer (Rigaku, DMAX 2200) with $2 \theta$ from $5^{\circ}$ to $60^{\circ}$ equipped with a Cu tube $(\lambda=0.15418 \mathrm{~nm})$ and a secondary monochromator. From the XRD parameters, the interlayer distances (d002) in the graphene were estimated using Bragg's Law ${ }^{28}$. The grain size (C) was calculated using the Scherrer equation ${ }^{29}$ following Equation 1.

$$
C=\frac{0.9 \lambda}{\beta \cos \theta}
$$

Here, $\beta$ is the line width at half height in radians and $\theta$ is the diffraction angle.

The interlamellar distance was estimated by the Bragg's law and the number of the stacked sheets by the DebyeScherrer equation. Likewise, the average size of crystallites (D) was determined from the (100) plane reflection ${ }^{30}$.
Carbon molecular bonding characteristics of GO and RGO were characterized by Raman spectrum performed at room temperature using Olympus microscope and an iH320 Jobin Yvon Spectrometer with a CCD (charged coupled device) detector, cooled by liquid nitrogen. Raman shift varied from 500 to $3000 \mathrm{~cm}^{-1}$. The excitation source was a $10 \mathrm{~mW}$ HeNe laser.

\subsubsection{Elemental analysis}

Elemental analysis was made in a LECO Truspec CHN equipped with detectors of infrared and of thermal conductivity. For the analysis, the samples were burned under an oxygen atmosphere (purity $99.99 \%$ ) at a temperature of $950{ }^{\circ} \mathrm{C}$.

\subsubsection{Electrical conductivities}

The electrical conductivities were made on pressed pellets of the $\mathrm{GO}$ and $\mathrm{RGO}$ (13 mm diameter, $<1 \mathrm{~mm}$ thick) with a home-made four-point collinear probe device. Keithley Instruments, model 236 and a Multimeter HP 34401 were used as current source and for measure of voltage, respectively.

\subsubsection{Hydrolytic degradation and swelling behavior}

The hydrolytic degradation experiments were carried out based on ASTM F1635-11. The CS membrane and CS/graphene nanocomposites samples cutt into 5-mm diameter disks was weighed (P0), dipped in tubes containing $5 \mathrm{~mL}$ of saline buffer solution (PBS), and incubated at $37^{\circ} \mathrm{C}$ temperature and $60 \mathrm{rpm}$-stirring (Shaker, MINITRON, model AG CH-4130). Each batch contained four specimens for each evaluated incubation time. The specimens were removed from PBS solution after 7, 14, 21,28 , and 70 days. They were carefully washed with deionized water, dried for 12 hours at $50^{\circ} \mathrm{C}$ to a constant weight, and then weighed (Pt) to determine mass loss. The percentages of mass loss were calculated from Equation 2.

$$
\% \text { weight loss }=[(\mathrm{P} 0-\mathrm{Pt}) / \mathrm{P} 0] \times 100
$$

The results were analyzed with two-way analysis of variance (ANOVA), followed by Bonferroni's test. Values of $p>0.05$ were considered statistically insignificant.

The swelling behavior of the membranes was studied by gravimetry. Two circular samples membranes were dried in a desiccator for 24 hours and weighed (S0) and placed in Falcon tubes containing $5 \mathrm{~mL}$ PBS solution $\left(\mathrm{pH} 7.4\right.$ at $37^{\circ} \mathrm{C}$ ) and remained during 1, 24 and 48 hours, at the end of this, the samples were removed of tubes, wiped gently with filter paper and weighed (Sf). The swelling degree (SW \%), was obtained through Equation 3.

$$
\begin{aligned}
& \% \mathrm{SW}=[(\mathrm{Sf}-\mathrm{S} 0)) / \mathrm{S} 0] * 100 \\
& \mathrm{Sf}=\text { mass of the swollen sample }(\mathrm{g}) \\
& \mathrm{S} 0=\text { mass of the dry sample }(\mathrm{g})
\end{aligned}
$$




\subsection{5 $\mathrm{pH}$ behavior}

Changes in the $\mathrm{pH}$ of the PBS solution (initial $\mathrm{pH}=$ 7.38) were monitored as a function of incubation time. To better evaluate the effects of incubation time, each group of samples is matched by the blank, in which only the PBS solution is stirred.

\subsubsection{Contact angle}

Water contact angle on the surface of the membrane was measured using a goniometer (Phoenix $300, \mathrm{SEO}$ ) at $25^{\circ} \mathrm{C}$. Prior to these measurements, all the membranes were vacuum dried and kept in a desiccator. Then, a piece of membrane $(1 \mathrm{~cm} \times 7 \mathrm{~cm})$ was adhered to clean slide for contact angle measurements. A $2 \mu \mathrm{L}$ of deionized water droplet was placed on the sample surface and droplet image was captured.
All the measurements were done within $10 \mathrm{~s}$ to minimize the errors due to evaporation losses ${ }^{31}$. A total of six measurements were taken at different locations for each membrane sample. The results were analyzed with one-way analysis of variance (ANOVA), followed by Bonferroni's test. Values of $p>0.05$ were considered statistically insignificant.

\section{Results and Discussion}

\section{$3.1 \mathrm{GO}$ and $R G O$}

Figure 1 shows the morphologies of the different carbonbased materials investigated in this study. FESEM shows that FINE raw graphite (Figure 1A) comprises compact stacks of graphene sheets; after oxidation, GO showed increased spacing between the sheets and a flat structure (Figure 1B).
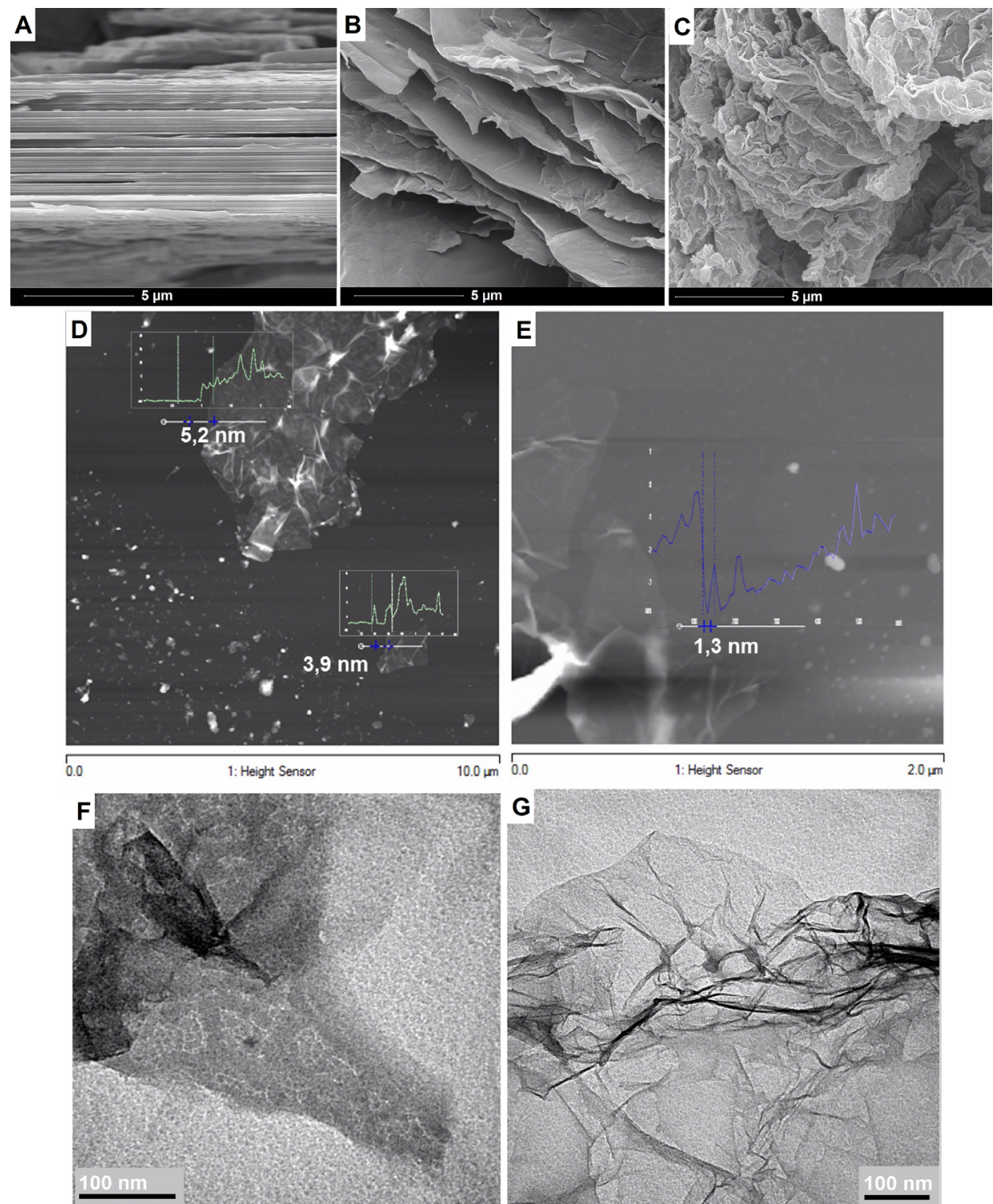

Figure 1. FESEM images of FINE (A), GO (B) and RGO (C). AFM images of GO (D) and RGO (E). TEM images of GO (F) and RGO (G). 
RGO (Figure 1C) revealed a typical rough morphology formed by corrugated sheets. AFM analyses indicated that the distance between the $\mathrm{Si}$ substrate and the GO surface is $\sim 5.9 \mathrm{~nm}$ (Figure 1D); and this value decreases to $1.3 \mathrm{~nm}$ for the RGO sample (Figure 1E) owing to the partial removal of oxygenated groups. The TEM images (Figure 1F-G) also indicate that RGO is more exfoliated and corrugated than GO.

Figure 2 shows XRD patterns of FINE raw graphite, GO, and RGO. The XRD pattern of FINE shows a dominant peak at $2 \theta=26^{\circ}$ that is typical of graphite. GO formation is confirmed by the absence of the peak at $2 \theta=26^{\circ}$ and the appearance of a diffraction peak at $11^{\circ}$ that is attributed to the increased layer spacing owing to the introduction of oxygen-containing groups into graphite ${ }^{32,33}$.

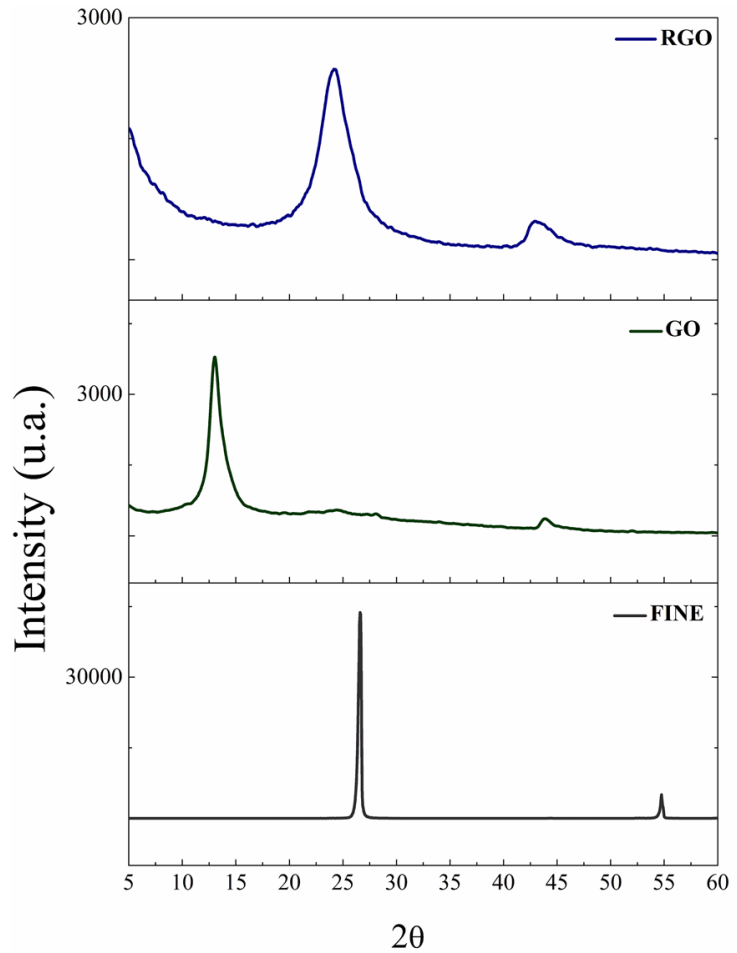

Figure 2. XRD patterns of FINE, GO and RGO.

Table 1 lists the XRD parameters. The thermal reduction process of GO to RGO decreased the interlayer distance (d002) between the graphene sheets owing to the partial elimination of oxygen functional groups. Moreover, a marked decrease in the grain size (C) compared to the raw graphite FINE was observed. The oxidation reaction also decreased the number of stacked layers.
The decrease in the average size of $\mathrm{C}$ and the number of estimated graphene layers suggested an efficient exfoliation process. The results obtained are in agreement with those observed in previous studies ${ }^{25,34}$.

The chemical oxidation process can result in prominent structural changes in the graphite lattice owing to the formation of different types of oxygen-containing groups at both the basal plane and at the edges ${ }^{35}$. Raman spectroscopy is commonly considered most appropriate for characterizing the structure of graphite materials ${ }^{36}$. Figure 3 shows the typical Raman spectra of raw graphite (FINE), GO and RGO. The characteristic $G$ and $D$ bands of graphene-based materials are observed at $1600 \mathrm{~cm}^{-1}$ and $1360 \mathrm{~cm}^{-1}$. These two bands are related to the graphitized structure and local defects/disorders, respectively. The intensity of the D band is a measure of the amount of disorder in graphene, because the activation of the $\mathrm{D}$ band is attributed to the breaking of the translational symmetry of the $\mathrm{C}=\mathrm{C} \mathrm{sp}^{2}$ bond. The $\mathrm{D}$ peak results from the existence of oxygen-containing functional groups of GO that are negligible in graphite (FINE) ${ }^{6,7}$. For GO and RGO, the $\mathrm{D}$ band becomes stronger and broader because of the higher level of disorder of the graphene layers ${ }^{37,38}$.

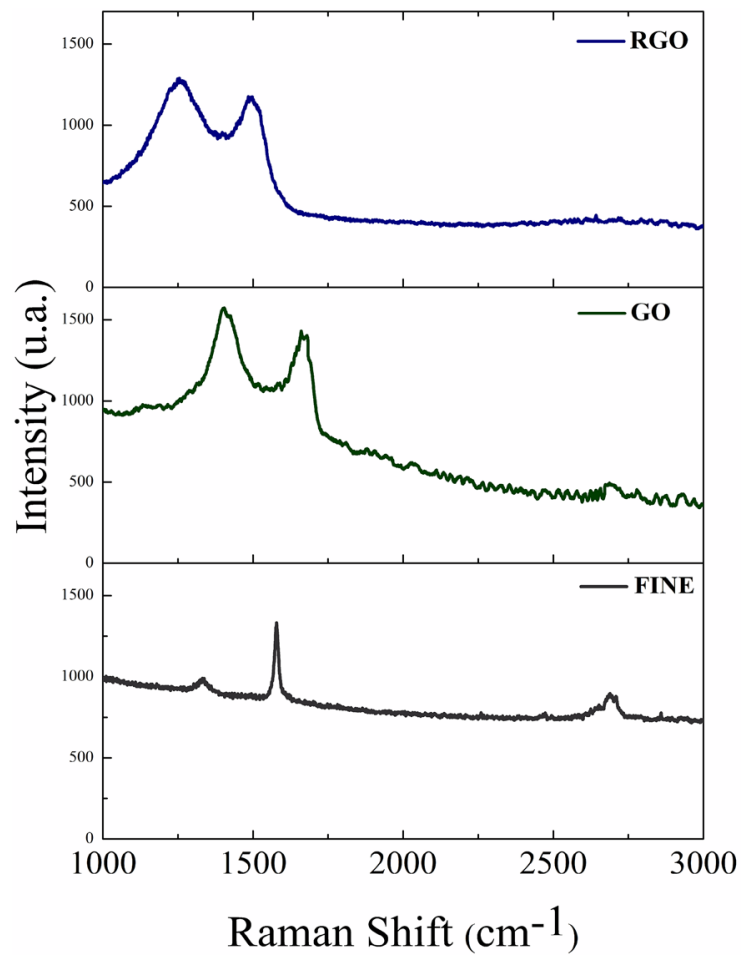

Figure 3. Raman spectra of FINE, GO and RGO.

Table 1. Data obtained from the XRD patterns for FINE, GO and RGO.

\begin{tabular}{ccccc}
\hline Sample & $2 \theta\left(^{\circ}\right)$ & $\mathrm{d}_{002}(\mathrm{~nm})$ & Grain size C $(\mathrm{nm})$ & Graphene stacks \\
\hline FINE & 26 & 0.33 & 30 & 90 \\
GO & 11 & 0.74 & 8 & 10 \\
RGO & 24 & 0.36 & 3 & 8 \\
\hline
\end{tabular}


According to Table 2, the elemental analysis and electrical conductivity data are consistent with the XRD results. A decrease in the amount of oxygen $(\% \mathrm{O})$ in the reduced sample can be seen when compared with the GO, indicating successful thermal reduction. The oxygen-functionalized graphene sheets show promise for several applications such as the preparation of composites in which these groups may play an important role in the interaction between graphene sheets and the polymeric matrix ${ }^{35}$. Table 2 indicates that the oxidation process of the FINE graphite decreased the electric conductivity; however, the reduction process very efficiently restored the $\mathrm{sp}^{2}$ network, which is responsible for electric conductivity.

\subsection{Nanocomposites}

Figure 4 shows the FESEM of the surface morphology of a pristine CS membrane and composites with GO and RGO loadings (Figure 4B-C). The membrane surface became more roughened with the addition of GO and RGO, indicating that they are coated by CS. Khan and Pokhrel reported similar results ${ }^{39,40}$.
The average membrane thickness is $46.6-51.9 \mu \mathrm{m}$, and the cross-section of the pristine CS membrane has smooth morphology (Figure 4D). However, the roughness increases with the addition of graphene [Figure 4E-F]. The FESEM images show that wrinkled graphene sheets are uniformly distributed in the polymer matrix. These results indicated that the oxidation of graphene introduced anchoring sites for forming an effective CS coating on graphene. RGO addition resulted in a more roughened membrane owing to the reduction of oxygenated groups that decrease the interaction between the filler and the matrix polymer ${ }^{41-43}$.

Studying swelling is important because it predicts how much the membrane will swell when in contact with biological fluids. Preventing large expansion of the membrane during its therapeutic action and avoiding possible compression of the tissues where it will be applied are important issues. The membrane's degree of swelling (\% SW) was evaluated in a phosphate-buffered saline (PBS) solution with incubation for up to $48 \mathrm{~h}$ (Figure 5). The degree of swelling is initially higher and then gradually decreases. However, there are some differences in the maximum swelling ratios.

Table 2. Elemental analysis and electrical conductivity for FINE, GO and RGO.

\begin{tabular}{cccc}
\hline \multirow{2}{*}{ Sample } & \multicolumn{2}{c}{ CHN } & Electrical conductivity (S/cm) \\
\cline { 2 - 4 } & $\% \mathrm{C}$ & $\% \mathrm{O}$ & 1200.0 \\
FINE & 98.3 & 1.7 & 3.1 \\
GO & 70.3 & 28.7 & 60.0 \\
\hline
\end{tabular}
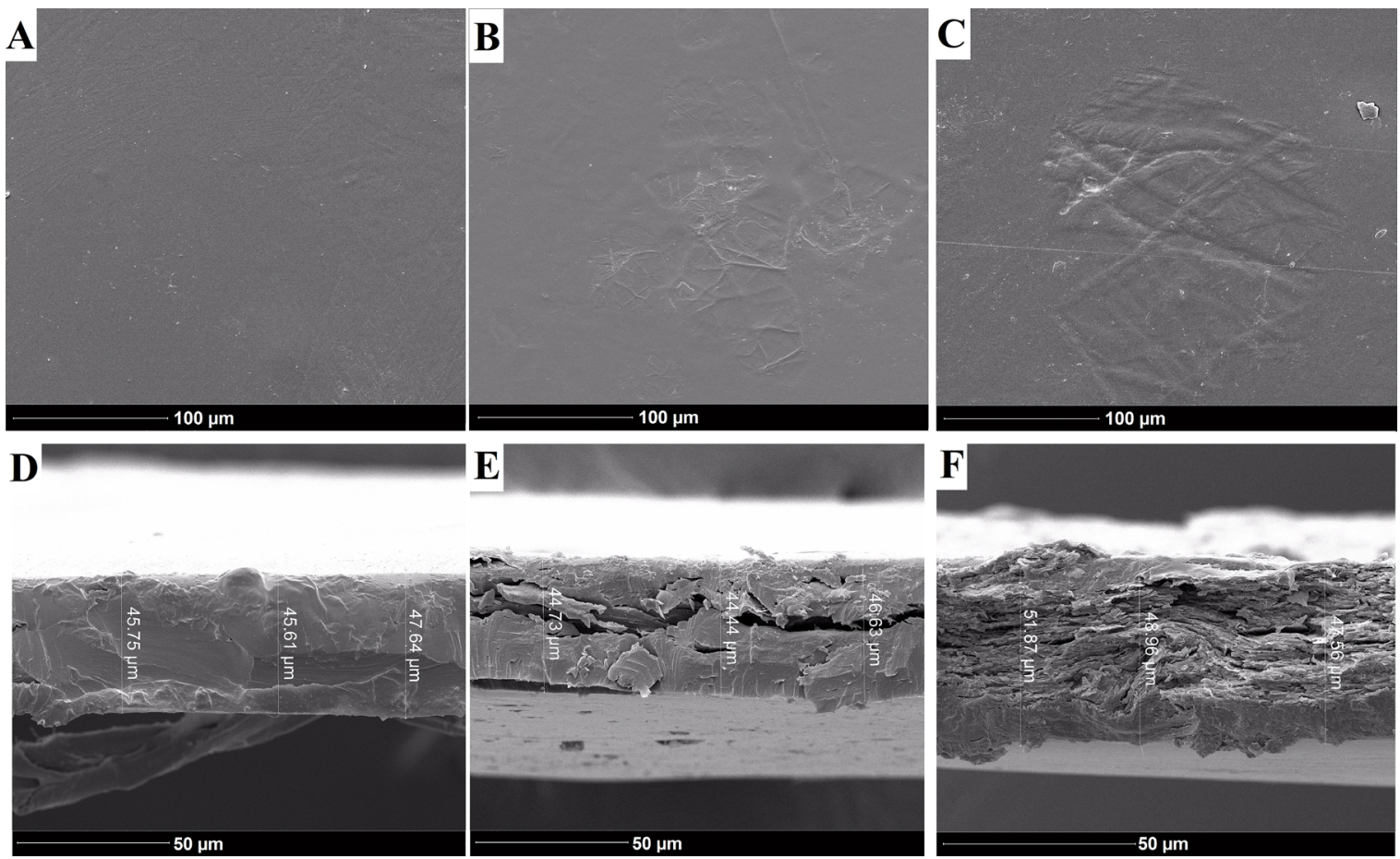

Figure 4. FESEM of the surface of CS (A), CS/GO3 (B) and CS/RGO3 (C). FESEM of the cross-section: CS (D), CS/GO3 (E) and CS/RGO3 (F). 
At $24 \mathrm{~h}$, the pristine CS membrane shows the maximum swelling degree (875\%) whereas the CS/graphene membranes show lower swelling degrees. These results can be explained considering that the charge interacts with the polymer and stabilizes the CS network and reduces the availability of hydrophilic groups ${ }^{15,44,45}$. Membranes with RGO (Figure 5B) presented lower \% SW (370\%) than membranes with GO (Figure 5A), probably because RGO nanosheets provide fewer hydrophilic groups to restrain the swelling of the CS/ RGO nanocomposites, leading to a lower swelling ratio ${ }^{44}$. These samples show rapid increase in PBS content and reach equilibrium within 48 hours.

The important characteristic of CS is its biodegradability. To evaluate the effect of the addition of GO and RGO in the polymer matrix, the membrane mass loss in the PBS solution (physiological $\mathrm{pH}$ ) was evaluated over a 70-day period. Figure 6 shows comparative results with a pure CS membrane and a CS membrane with graphene-based fillers.

Figure 6A shows that the CS/GO 0.5 membrane was the only one that presented a lower mass loss percentage compared with the CS membrane within 7 days.
CS membranes filled with GO showed no differences in the mass loss behavior in relation to the pristine CS control at intervals evaluated up to 28 days. A statistically significant influence was observed for the $\mathrm{CS} / \mathrm{GO}$ composites after 70 days of incubation (27\%) compared with the mass loss percentage of the pure CS sample (20\%), demonstrating that GO does not greatly increase the mass loss of the membranes. Similarly, nanocomposite films with RGO (Figure 6B) studied at intervals of 7, 14, 21, and 28 days did not show significant differences in the mass loss percentage in relation to the control CS membrane. These results indicates that the increase in the percentage of RGO in the CS matrix does not accelerate the degradation time of CS membranes in the PBS solution.

The changes in the $\mathrm{pH}$ of the $\mathrm{PBS}$ solution (initial $\mathrm{pH}=7.38$ ) were monitored as a function of the degradation time. There were no significant changes in the $\mathrm{pH}$ values of the PBS solutions for incubation periods of up to 70 days. These results show that the normal physiological condition ${ }^{46}$ was maintained during the incubation time even in graphene/ CS-based membranes.
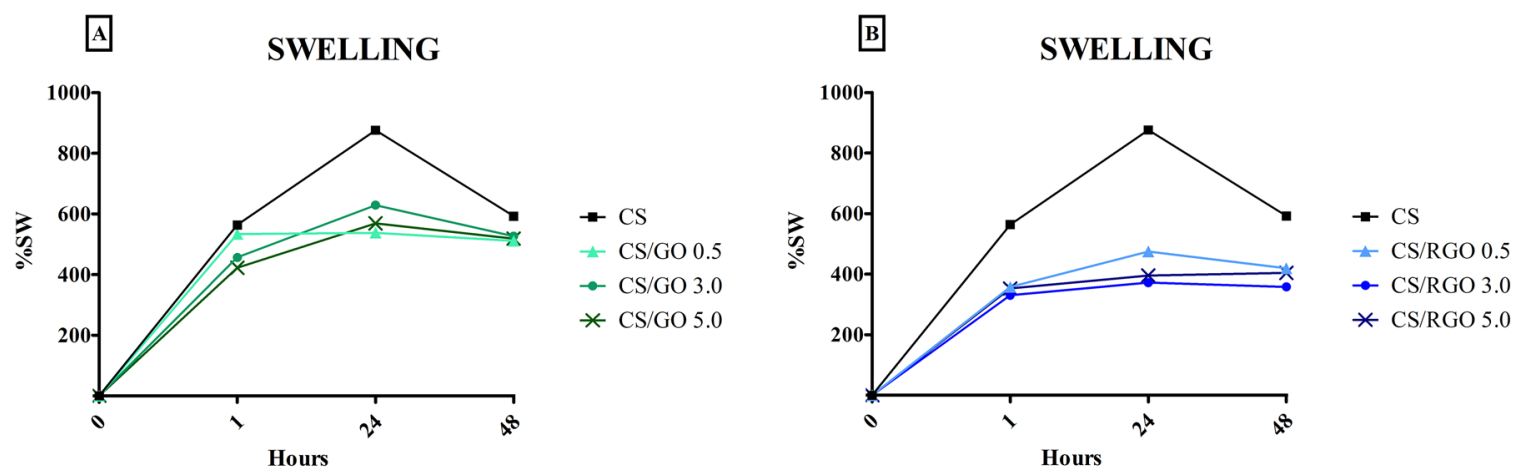

Figure 5. Comparative graph of Swelling Degree (\%SW) between pristine and filled chitosan membranes: (A) CS/GO and (B) CS/RGO.
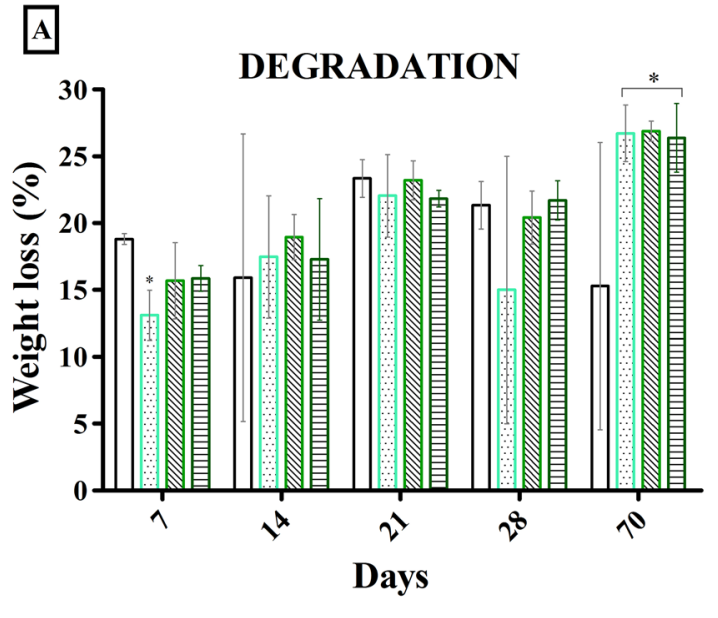

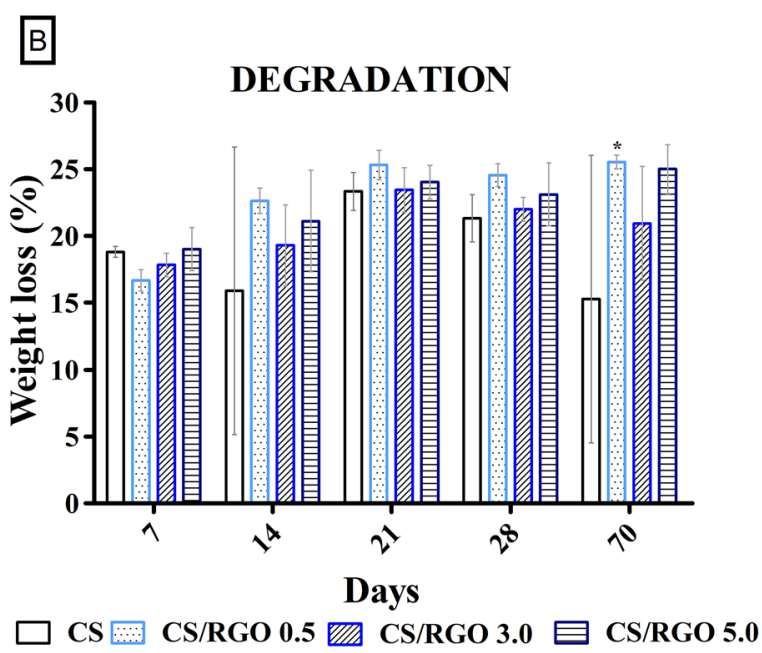

Figure 6. Comparative weight loss results with pristine CS membranes and CS membranes with graphene based fillers: CS/GO (A) and $\mathrm{CS} / \mathrm{RGO}(\mathrm{B}) .{ }^{*} \mathrm{p}<0,05$. 
The water contact angle (WCA) of the prepared membranes was measured to investigate the hydrophilicity changes after the addition of GO and RGO to the CS matrix. The WCA data provide information about the relative degree of surface hydrophilicity of all membranes; the smaller the contact angle, the higher is the hydrophilicity ${ }^{31}$. Figure 7 shows the WCA results. These results show no significant difference $(\mathrm{P}<0.05)$ between the nanocomposites and the pristine CS membrane, indicating that graphene species do not have a negative effect on the CS hydrophilicity. This result was desirable because the hydrophilic membrane has potential applications in biomedicine ${ }^{47}$.

\section{Conclusions}

GO and RGO containing 8-10 stacked graphene layers were produced using Staudenmaier's modified method. It was possible to produce GO with low density of defects for applications such as biopolymer composites. This study reports a facile method to obtain CS nanocomposites with a few graphene layers. The addition of GO and RGO to the CS matrix did not accelerate the composite degradation process during the 70 days of incubation and did not change the $\mathrm{pH}$. The presence of functional groups in the fillers decreases the swelling process compared to that in the pristine CS membrane; however, it did not modify the surface hydrophilicity. These results established that the addition of GO and RGO in the CS matrix is an important strategy for preparing composites for applications in therapeutics.

\section{CS \\ $\mathrm{WCA}=91.12 \pm 4,42^{\circ}$}

\section{CS/GO 0.5}

$\mathrm{WCA}=93.20 \pm 1.73^{\circ}$

\section{Acknowledgments}

This study was financed in part by the Coordenação de Aperfeiçoamento de Pessoal de Nível Superior Brasil (CAPES) - Finance Code 001. The authors are also grateful the Nacional de Grafite Ltda, for supplying the Grafine 99200 from which the GO and RGO were obtained.

\section{References}

1. International Union of Pure and Applied Chemistry (IUPAC), McNaught AD, Wilkinson A. Graphene layer - Compendium of Chemical Terminology. $2^{\text {nd }}$ ed. Oxford: Blackwell Scientific Publications; 2014.

2. Ghuge AD, Shirode AR, Kadam VJ. Graphene: a comprehensive review. Current Drug Targets. 2017;18(6):724-33.

3. Liu J, Qiao Y, Guo CX, Lim S, Song H, Li CM. Graphene/ carbon cloth anode for high-performance mediatorless microbial fuel cells. Bioresource Technology. 2012;114:27580.

4. Kausar A. Polymer/graphene nanocomposite: preparation to application. American Journal of Polymer Science and Engineering. 2016;4(1):111-22.

5. Grande CD, Mangadlao J, Fan J, De Leon A, DelgadoOspina J, Rojas JG, et al. Chitosan cross-linked graphene oxide nanocomposite films with antimicrobial activity for application in food industry. Macromolecular Symposia. 2017;374(1):1-8.

\section{CS/GO 3}

$\mathrm{WCA}=90.63 \pm 4.20^{\circ}$

\section{CS/GO5}

$\mathrm{WCA}=93.00 \pm 3.21^{\circ}$
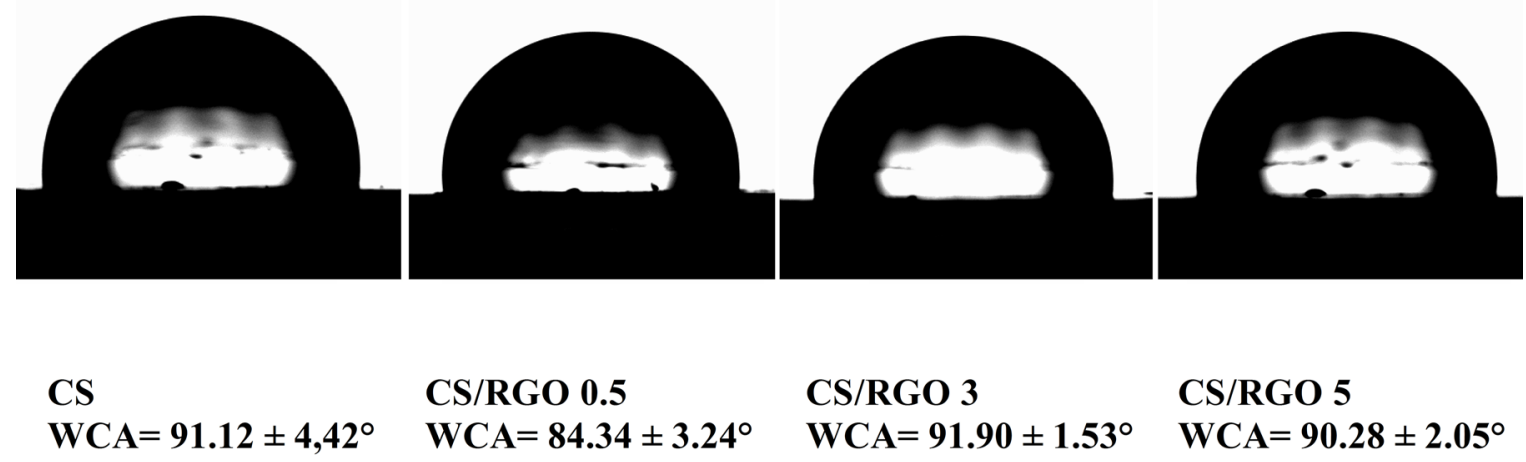

CS/RGO 0.5

$\mathrm{WCA}=84.34 \pm 3.24^{\circ}$

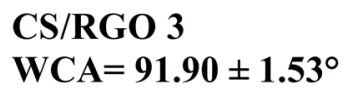

CS/RGO 5

$\mathrm{WCA}=\mathbf{9 0 . 2 8} \pm \mathbf{2 . 0 5 ^ { \circ }}$
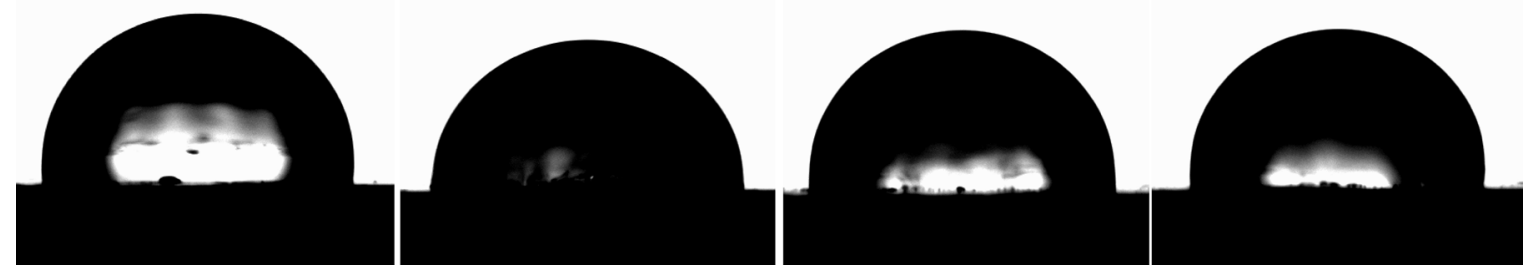

Figure 7. Water contact angle of chitosan membranes as a function of $w t \%$ of graphene fillers. ${ }^{*} \mathrm{p}<0,05$ 
6. Huang Q, Li G, Chen M, Dong S. Graphene oxide functionalized O-(carboxymethyl)-chitosan membranes: fabrication using dialysis and applications in water purification. Colloids and Surfaces A: Physicochemical Engineering Aspects. 2018;554:27-33.

7. Han SJ, Lee H Il, Jeong HM, Kim BK, Raghu AV, Reddy KR. Graphene modified lipophilically by stearic acid and its composite with low density polyethylene. Journal of Macromolecular Science: Part B: Physics. 2014;53(7):1193-204.

8. Bhuyan MSA, Uddin MN, Islam MM, Bipasha FA, Hossain SS. Synthesis of graphene. International Nano Letters. 2016;6:65-83.

9. Shah R, Kausar A, Muhammad B, Shah S. Progression from graphene and graphene oxide to high performance polymerbased nanocomposite: a review. Polymer-Plastics Technology and Engineering. 2015;54(2):173-83.

10. Hegab HM, Elmekawy A, Zou L, Mulcahy D, Saint CP, Ginic-Markovic M. The controversial antibacterial activity of graphene-based materials. Carbon. 2016;105:362-76.

11. Haghdadeh $P$, Ghaffari M, Ramezanzadeh B, Bahlakeh G, Saeb MR. The role of functionalized graphene oxide on the mechanical and anti-corrosion properties of polyurethane coating. Journal of the Taiwan Institute of Chemical Engineers. 2018;86:199-212.

12. Ji H, Sun H, Qu X. Antibacterial applications of graphene-based nanomaterials: recent achievements and challenges. Advanced Drug Delivery Reviews. 2016;105(Pt B):176-89.

13. Khorshidi B, Hajinasiri J, Ma G, Bhattacharjee S, Sadrzadeh M. Thermally resistant and electrically conductive PES/ITO nanocomposite membrane. Journal of Membrane Science. 2016;500:151-60.

14. Castrillón SRV, Perreault F, Faria AF, Elimelech M. Interaction of graphene oxide with bacterial cell membranes: insights from force spectroscopy. Environmental Science and Technology Letters. 2015;2(4):112-7.

15. Zuo PP, Feng HF, Xu ZZ, Zhang LF, Zhang YL, Xia W, et al. Fabrication of biocompatible and mechanically reinforced graphene oxide-chitosan nanocomposite films. Chemistry Central Journal. 2013;7(1):39.

16. Terzopoulou Z, Kyzas GZ, Bikiaris DN. Recent advances in nanocomposite materials of graphene derivatives with polysaccharides. Materials (Basel). 2015;8(2):652-83.

17. Sá ML, Carvalho ÉM, Calvacante J, Araque LM, Reis Sobrinho JF, Barbosa R, et al. Biodegradation of Poly (3-hydroxybutyrate) /Eggshellsystems. Materials Research. 2018;21(4):e20170792. DOI: https:/doi.org/10.1590/1980-5373-mr-2017-0792

18. Sayyar S, Murray E, Gambhir S, Spinks G, Wallace GG. Synthesis and characterization of covalently linked graphene/ chitosan composites. JOM. 2016;68(1):384-90.

19. Gonçalves RP, Ferreira WH, Gouvêa RF, Andrade CT. Effect of chitosan on the properties of electrospun fibers from mixed poly(vinyl alcohol)/chitosan solutions. Materials Research. 2017;20(4):984-93.

20. Depan D, Shah JS, Misra RDK. Degradation mechanism and increased stability of chitosan-based hybrid scaffolds crosslinked with nanostructured carbon: process-structure-functional property relationship. Polymer Degradation and Stability. 2013;98(11):2331-9.
21. Suhas DP, Aminabhavi TM, Jeong HM, Raghu AV. Hydrogen peroxide treated graphene as an effective nanosheet filler for separation application. RSC Advances. 2015;5(122):100984-95.

22. Hu H, Wang X, Wang J, Liu F, Zhang M, Xu C. Microwaveassisted covalent modification of graphene nanosheets with chitosan and its electrorheological characteristics. Applied Surface Science. 2011;257:2637-42.

23. Zhang HP, Luo XG, Lin XY, Lu X, Tang Y. The molecular understanding of interfacial interactions of functionalized graphene and chitosan. Applied Surface Science. 2016;360(Pt B):715-21.

24. Bagheripour E, Moghadassi AR, Hosseini SM, Van Der Bruggen B, Parvizian F. Novel composite graphene oxide/chitosan nanoplates incorporated into PES based nanofiltration membrane: chromium removal and antifouling enhancement. Journal of Industrial and Engineering Chemistry. 2018;62:311-20.

25. Pavoski G, Maraschin T, Fim FC, Balzaretti NM, Galland GB, Moura CS, et al. Few layer reduced graphene oxide: evaluation of the best experimental conditions for easy production. Materials Research. 2017;20(1):53-61.

26. Herrera-Alonso M, Abdala AA, McAllister MJ, Aksay IA, Prud'homme RK. Intercalation and stitching of graphite oxide with diaminoalkanes. Langmuir. 2007;23(21):10644-9.

27. Campos MGN, Mei LHI, Santos Junior AR. Sorbitol-plasticized and neutralized chitosan membranes as skin substitutes. Materials Research. 2015;18(4):781-90.

28. Aladekomo JB, Bragg RH. Structural transformations induced in graphite by grinding: analysis of $002 \mathrm{X}$-ray diffraction line profiles. Carbon. 1990;28(6):897-906.

29. Debye P, Scherrer P. Werk Übergeordnetes Werk. Nachr Ges Wiss Göttingen, Math-physik Klasse. 1918;2:101-20.

30. Aguilar-Bolados H, Contreras-Cid A, Yazdani-Pedram M, Acosta-Villavicencio G, Flores M, Fuentealba P, et al. Synthesis of fluorinated graphene oxide by using an easy one-pot deoxyfluorination reaction. Journal of Colloid and Interface Science. 2018;524:219-26.

31. Suhas DP, Aminabhavi TM, Raghu AV. Para-toluene sulfonic acid treated clay loaded sodium alginate membranes for enhanced pervaporative dehydration of isopropanol. Applied Clay Science. 2014;101:419-29.

32. Li H, Zhu Y, Mao Z, Gu J, Zhang J, Qian Y. Synthesis and characterization of carbon fibrils formed by stacking graphite sheets of nanometer thickness. Carbon. 2009;47(1):328-30.

33. Huh SH. X-ray diffraction of multi-layer graphenes: instant measurement and determination of the number of layers. Carbon. 2014;78:617-21.

34. Nisar M, Bergmann CP, Geshev J, Quijada R, Maraschin T, Basso NRS, et al. Synthesis of high-density polyethylene/ rGO-CNT-Fe nanocomposites with outstanding magnetic and electrical properties. Journal of Applied Polymer Science. 2017;134(40):1-7.

35. Shen L, Zhang L, Wang K, Miao L, Lan Q, Jiang K, et al. Analysis of oxidation degree of graphite oxide and chemical structure of corresponding reduced graphite oxide by selecting differentsized original graphite. RSC Advances. 2018;8(31):17209-17. 
36. Li ZQ, Lu CJ, Xia ZP, Zhou Y, Luo Z. X-ray diffraction patterns of graphite and turbostratic carbon. Carbon. 2007;45(8):1686-95.

37. Wu JB, Lin ML, Cong X, Liu HN, Tan PH. Raman spectroscopy of graphene-based materials and its applications in related devices. Chemical Society Reviews. 2018;47(5):1822-73.

38. Ferrari AC, Basko DM. Raman spectroscopy as a versatile tool for studying the properties of graphene. Nature Nanotechnology. 2013;8(4):235-46

39. Khan YH, Islam A, Sarwar A, Gull N, Khan SM, Munawar MA, et al. Novel green nano composites films fabricated by indigenously synthesized graphene oxide and chitosan. Carbohydrate Polymers. 2016;146:131-8.

40. Pokhrel S, Yadav PN, Adhikari R. Applications of chitin and chitosan in industry and medical science: a review. Nepal Journal of Science and Technology. 2016;16(1):99-104.

41. Yenier Z, Seki Y, Şen I, Sever K, Mermer Ö, Sarikanat M. Manufacturing and mechanical, thermal and electrical characterization of graphene loaded chitosan composites. Composites Part B: Engineering. 2016;98:281-7.

42. Kim KT, Dao TD, Jeong HM, Anjanapura RV, Aminabhavi TM. Graphene coated with alumina and its utilization as a thermal conductivity enhancer for alumina sphere/thermoplastic polyurethane composite. Materials Chemistry and Physics. 2015;153:291-300.
43. Son DR, Raghu AV, Reddy KR, Jeong HM. Compatibility of thermally reduced graphene with polyesters. Journal of Macromolecular Science: Part B: Physics. 2016;55(11):1099110.

44. Zhang Y, Zhang M, Jiang H, Shi J, Li F, Xia Y, et al. Bio-inspired layered chitosan/graphene oxide nanocomposite hydrogels with high strength and $\mathrm{pH}$-driven shape memory effect. Carbohydrate Polymers. 2017;177:116-25.

45. Zhang S, Zou Y, Wei T, Mu C, Liu X, Tong Z. Pervaporation dehydration of binary and ternary mixtures of n-butyl acetate, n-butanol and water using PVA-CS blended membranes. Separation and Purification Technology. 2017;173:314-22.

46. Zhao X, Wei Z, Zhao Z, Miao Y, Qiu Y, Yang W, et al. Design and development of graphene oxide nanoparticle/chitosan hybrids showing $\mathrm{pH}$-sensitive surface charge-reversible ability for efficient intracellular doxorubicin delivery. ACS Applied Materials and Interfaces. 2018;10(7):6608-17.

47. Tavakoli M, Karbasi S, Bakhtiari SSE. Evaluation of physical, mechanical, and biodegradation of chitosan/graphene oxide composite as bone substitutes. Polymer-Plastics Technology and Materials. 2019;59(4):430-40. 